\title{
Parental smoking and the nutrient intake and food choice of British teenagers aged
} 16-17 years

\author{
H F Crawley, D While
}

\begin{abstract}
Study objective - To examine the association between parental smoking habits and the nutrient intake and food choice of teenagers aged 16-17 years, allowing for differences in teenage smoking and the social class and regional distribution of the participants.
\end{abstract}

Design - Data were collected from the 1970 longitudinal birth cohort, cross-sectionally at 16-17 years. The smoking habits of teenagers were evaluated from a questionnaire completed by the subjects themselves, and the smoking habits of parents by interview. The nutrient and food intakes of teenagers were quantitatively assessed using a four day unweighed dietary diary. Setting - The participants were distributed throughout Britain.

Participants - A subsample of 1222 males and 1735 females was isolated from respondents to the 1970 birth cohort 16-17 year data collection sweep undertaken in 1986-87.

Main results - Parental smoking habits were associated with different dietary patterns among teenagers regardless of whether the teenagers themselves smoked. Dietary differences noted were similar to those observed previously among smokers, with lower intakes of fibre, vitamin C, vitamin $E$, folates, and magnesium in particular reported among both males and females in households where parents were smokers. These lower intakes were associated with lower intakes of fruit juices, wholemeal bread, and some vegetables.

Statistics, Operational Research and Probability Methods Research Group (STORM),

The University

North London,

Holloway Road,

London N7 8DB

H F Crawley

The CRC Education and Child Studies Research Group, Department of Public Health and Epidemiology, The University Manchester, Oxford Road, Manchester M13 9PT D While

Correspondence to: Dr H Crawley.

Accepted for publication November 1995
Conclusion - Teenagers who lived with parents who smoked had different nutrient and food intakes to those with non-smoking parents, and teenagers exposed to parental smoking appeared to have similar dietary patterns to teenagers who themselves smoked.

( $\mathcal{F}$ Epidemiol Community Health 1996;50:306-312)

The dietary habits of adult smokers in Britain have been widely investigated, ${ }^{1-5}$ and it has been established that smokers generally choose diets that are different from those of nonsmokers. Smokers have been reported to consume diets lower in dietary fibre, polyunsaturated fatty acids, and vitamins $A$ and $C$, and higher in alcohol, than non-smokers. Smokers seem to eat more fat spreads, chips, processed meats, and fried foods and to drink more coffee and alcoholic beverages than nonsmokers, but to consume smaller amounts of fresh fruit and vegetables, cakes, biscuits, and pastries. The role of smoking on the nutrient and food intake of teenagers aged 16-17 years has also recently been investigated. ${ }^{6}$ Teenage smokers reported similar dietary patterns to those of adult smokers, and the association between smoking and differences in dietary habits was stronger among teenage girls Among regular teenage smokers in this study, allowing for differences in age, region, social class, and dieting habits, both male and female smokers consumed more alcohol and less fibre and vitamin $\mathrm{C}$ than occasional or never smokers. Female smokers also had lower intakes of selenium, carotene, and folates. These differences were reflected in the different food choices made by teenage smokers, who generally consumed fewer puddings, biscuits, breakfast cereals, wholemeal breads, fruit, and fruit juices, but more alcoholic drinks, coffee, and chips.

Although teenagers at $16-17$ years are likely to be making many independent food choices, many will still rely on food provided within the family, and will be influenced by parental food choices. It has been suggested that teenagers are faced with a dietary duality, and manage this by following the parental lead on food eaten in the home, but in company with peers opt for foods more commonly associated with adolescence such as confectionery, fried foods, and take away meals. ${ }^{7}$ It could therefore be suggested that a smoking parent is likely to influence at least part of the diet of their teenager, regardless of whether that teenager is a smoker themselves. Investigations into the choice of food within the home in a two parent family have shown that women often defer to the taste preferences of the man of the house, and the effect of parental smoking may therefore also depend on which parent is a smoker. This study aimed to investigate the role of parental smoking on the nutrient and food intakes of teenagers at 16-17 years.

\section{Methods}

\section{PARTICIPANTS}

Participants in this study were isolated from respondents to the 1970 longitudinal birth cohort (BCS70) 16-17 year survey in 1986-87. BCS70 has monitored the health, education, and lifestyle of all children born in Britain between 5 and 11 April $1970,{ }^{9}$ and at 16-17 years approximately 12000 of the original 
15000 cohort members responded to some aspect of the follow up survey. ${ }^{10}$ In order to be included in the analyses for this study, respondents needed to have returned a satisfactorily completed four day dietary diary, and to have responded fully to a self completion questionnaire on smoking habits. In addition, data on parental smoking habits from the parental interview were required. Of the almost 5000 dietary diaries returned, 4760 were coded for analysis (other returned diaries were blank, incomplete, or inadequately detailed). Of these, 4375 respondents could be classified by social class, based on the occupation of the head of household using the Registrar General's job classification of occupations. ${ }^{11}$ This sample has been described elsewhere ${ }^{12}$ and with the exception of a low response rate in London, has been shown to be moderately representative of the British population both regionally and by social class. Matched data on teenage and parental smoking habits were available for 2957 $(68 \%)$ of the dietary diary respondents with social class data $($ male $=1222$, female $=1735)$. This sample does not therefore represent a random sample of teenagers, since those included were those where both the teenager and their parents consistently responded to a number of survey instruments. No significant differences in the population distributions by region and social class were noted between the participating sample in this study and the 1418 (32\%) respondents who completed a diary but for whom insufficient data were available on teenage and parental smoking. Despite limitations in the selection of participants in this study, this remains a useful sample for investigation since it is large, unusually includes information on parental habits as well as detailed food and nutrient intake data from the participants, and since the potential exists for continued longitudinal study.

\section{DATA COLLECTION METHODS}

Dietary data were collected in four day unweighed dietary diaries, completed from Friday to Monday of one week, and were quantitatively coded to provide data on the intakes of all nutrients (excluding estimates of fatty acid intakes, but including intakes of individual sugars and fibre expressed as non-starch polysaccharides (NSP)) and 80 distinct food groups. A detailed account of the dietary diary analysis, nutrient database used, and a discussion of the validity of the dietary diary method have been presented elsewhere. ${ }^{12}$ Briefly, unweighed dietary diaries were quantitatively coded using relevant information provided in the dietary records and standard food portion sizes. ${ }^{13} \mathrm{~A}$ nutrient database was specifically compiled for use in this study from a variety of published sources and from data obtained from The Ministry of Agriculture, Fisheries and Food (B Holland, personal communication) which included many items not available in standard food tables at that time but of importance in teenage diets, such as burgers, kebabs, vegetarian dishes, ethnic meals, and savoury snacks. The unweighed method of dietary data collection is economical in cost and researcher time when large numbers of individuals are to be studied, and has been suggested as a more accurate representation of eating habits than a one day recall or diet history. ${ }^{14}$ While the estimation of food weights remains a source of error, weighed intakes have frequently been shown to under estimate energy intake. ${ }^{15}$ A comparison of reported energy intakes to estimated basal metabolic rate measurements can be used to assess the validity of reported energy intakes, and in this study the unweighed diary method gave appropriate energy intake values among non-dieting teenagers. ${ }^{12}$

Teenage smoking data were collected via a self completion questionnaire and smoking habits were categorised from a number of questions relating to current and past smoking experience. Since the data relied on the teenager adequately understanding and completing the questionnaire, a consistent response to a number of questions on smoking habit was required to minimise the problems of reporting and coding errors. Smokers were initially categorised as never smokers, ex-smokers, experimental smokers (have tried a cigarette at least once), occasional smokers ( $<1$ cigarette per week), and regular smokers ( $>1$ cigarette per week). ${ }^{16}$ In order to provide samples of adequate size for data analysis and to preserve the never smoked and regular smoking categories, ex-smokers, experimental, and occasional smokers were grouped together as occasional smokers, a standard measure for this age group determined by Beverley. ${ }^{16}$ Parents were classified as either smokers or nonsmokers, based on data collected by home interview with a health visitor, and within the household smoking habits were defined as neither parent smoking, mother only smoking, father only smoking and both parents smoking. Respondents from single parent families were excluded from these analyses. Since the data collected on smoking habits were not validated biochemically, it is possible that a number of individuals did not accurately report smoking habits; however, studies which have compared self reported data with saliva cotinine measurements have shown good agreement in teenage populations. ${ }^{17}$

\section{STATISTICAL METHODS}

Differences between the participant group and the dietary diary responders who did not provide adequate data on teenage and parental smoking were investigated using three way contingency tables in the statistical package GLIM. ${ }^{18}$ Differences between the explanatory variables age, region, and social class in relation to parental smoking habits were investigated using cross tabulations and the $\chi^{2}$ test in SPSSX version 4.0. ${ }^{19}$ To describe the relationships between parental smoking and nutrient and food intakes, generalised linear models were fitted using the GLIM software package, to explain differences in nutrient and food intake using the explanatory variable of parental smoking, with social class, regional distribution, and total 
Table 1 Profile of study sample males by parental smoking habits

\begin{tabular}{|c|c|c|c|c|c|}
\hline & $\begin{array}{l}\text { All males } \\
(n=1222)\end{array}$ & $\begin{array}{l}\text { Neither parent smokes } \\
(n=593)\end{array}$ & $\begin{array}{l}\text { Mother only smokes } \\
(n=123)\end{array}$ & $\begin{array}{l}\text { Father only smokes } \\
(n=260)\end{array}$ & $\begin{array}{l}\text { Both parents smoke } \\
(n=246)\end{array}$ \\
\hline$\%$ aged $16 y$ & $70 \cdot 5$ & $70 \cdot 5$ & $70 \cdot 7$ & $69 \cdot 2$ & $72 \cdot 0$ \\
\hline $\begin{array}{l}\text { Never smokers (\%) } \\
\text { Regular smokers (\%) }\end{array}$ & $\begin{array}{l}44 \cdot 6 \\
16 \cdot 0\end{array}$ & $\begin{array}{l}49 \cdot 1^{*} \\
10 \cdot 9^{*}\end{array}$ & $\begin{array}{l}41 \cdot 5 \\
21 \cdot 1\end{array}$ & $\begin{array}{l}37 \cdot 7 \\
19 \cdot 2\end{array}$ & $\begin{array}{l}43 \cdot 9 \\
22 \cdot 4\end{array}$ \\
\hline $\begin{array}{l}\text { Region (\%): } \\
\text { South } \\
\text { Midlands/Wales } \\
\text { North } \\
\text { Scotland }\end{array}$ & $\begin{array}{l}31 \cdot 3 \\
32 \cdot 9 \\
25 \cdot 8 \\
10 \cdot 0\end{array}$ & $\begin{array}{l}34 \cdot 2 \dagger \\
34 \cdot 2 \\
23 \cdot 3 \\
8 \cdot 3 \dagger\end{array}$ & $\begin{array}{l}29 \cdot 3 \\
25 \cdot 2 \\
33 \cdot 3 \\
12 \cdot 2\end{array}$ & $\begin{array}{l}29 \cdot 6 \\
33 \cdot 5 \\
25 \cdot 0 \\
11 \cdot 9\end{array}$ & $\begin{array}{l}29 \cdot 7 \\
31 \cdot 7 \\
27 \cdot 6 \\
11 \cdot 0\end{array}$ \\
\hline Head of household non-manual (\%) & $49 \cdot 1$ & $59 \cdot 1^{*}$ & $39 \cdot 0$ & $46 \cdot 5$ & $33 \cdot 7$ \\
\hline
\end{tabular}

* Significant difference between "neither parent smokes" group and "either" or "both parents smoke" groups as $\chi^{2}$ statistic (p<0.001).

† Significant difference between "neither parent smokes" group and "either" or "both parents smoke" groups as $\chi^{2}$ statistic (p<0.05).

Table 2 Profile of study sample females by parental smoking habits

\begin{tabular}{|c|c|c|c|c|c|}
\hline & $\begin{array}{l}\text { All females } \\
(n=1735)\end{array}$ & $\begin{array}{l}\text { Neither parent smokes } \\
(n=809)\end{array}$ & $\begin{array}{l}\text { Mother only smokes } \\
(n=174)\end{array}$ & $\begin{array}{l}\text { Father only smokes } \\
(n=363)\end{array}$ & $\begin{array}{l}\text { Both parents smoke } \\
(n=389)\end{array}$ \\
\hline$\%$ aged $16 y$ & $69 \cdot 2$ & $69 \cdot 1$ & $70 \cdot 7$ & $68 \cdot 9$ & $64 \cdot 8$ \\
\hline $\begin{array}{l}\text { Never smokers (\%) } \\
\text { Regular smokers (\%) }\end{array}$ & $\begin{array}{l}41 \cdot 3 \\
19 \cdot 8\end{array}$ & $\begin{array}{l}50 \cdot 6^{*} \\
12 \cdot 4^{*}\end{array}$ & $\begin{array}{l}32 \cdot 2 \\
24 \cdot 7\end{array}$ & $\begin{array}{l}36 \cdot 1 \\
19 \cdot 0\end{array}$ & $\begin{array}{l}34 \cdot 7 \\
31 \cdot 6\end{array}$ \\
\hline $\begin{array}{l}\text { Region }(\%): \\
\text { South } \\
\text { Midlands/Wales } \\
\text { North } \\
\text { Scotland }\end{array}$ & $\begin{array}{r}32 \cdot 7 \\
28 \cdot 9 \\
29 \cdot 0 \\
9 \cdot 4\end{array}$ & $\begin{array}{l}37 \cdot 9 \dagger \\
26 \cdot 3 \\
28 \cdot 4 \\
7 \cdot 3 \dagger\end{array}$ & $\begin{array}{l}27 \cdot 0 \\
29 \cdot 3 \\
31 \cdot 0 \\
12 \cdot 6\end{array}$ & $\begin{array}{l}30 \cdot 6 \\
35 \cdot 8 \\
23 \cdot 4 \\
10 \cdot 2\end{array}$ & $\begin{array}{l}24 \cdot 9 \\
28 \cdot 5 \\
35 \cdot 0 \\
11 \cdot 6\end{array}$ \\
\hline Head of household non-manual (\%) & $47 \cdot 5$ & $54 \cdot 0^{*}$ & $39 \cdot 1$ & $50 \cdot 1$ & $34 \cdot 4$ \\
\hline
\end{tabular}

* Significant difference between "neither parent smokes" group and "either" or "both parents smoke" groups as $\chi^{2}$ statistic (p<0.001).

† Significant difference between "neither parent smokes" group and "either" or "both parents smoke" groups as $\chi^{2}$ statistic (p<0.05).

energy intake as covariates as appropriate. Using the concepts of parsimony and goodness of fit, the most appropriate models were selected which best explained the largest proportion of variability in the data set. To test the significance of differences between models, the $\mathbf{F}$ statistic was calculated and significance was observed using a $\chi^{2}$ table. In GLIM it is possible to choose an appropriate error choice rather than approximating a normal distribution by data transformation. For energy, protein, non-starch polysaccharides (NSP), carbohydrate, calcium, copper, zinc, and selenium intakes, normal errors were used and for alcohol, iron, iodine, vitamin C, thiamin, riboflavin, folates, retinol, and carotene Poisson errors were used with the identity link.

Intakes of foods could not be modelled directly using one distribution since there were a number of non-consumers for each food group. In order to establish whether differences in food intakes were due to the non-consumption of the food group or to a smaller amount being consumed, food intakes were modelled in two stages. In the first, the number of consumers of each food were compared, using log-linear models in GLIM with a Poisson data distribution. Secondly, examining the number of consumers in the group only, models were created which best explained the variations in intake among consumers. Food group intakes among consumers were modelled using the Poisson distribution and identity link. In both cases allowances were made for differences in region and social class.

\section{Results}

Overall, $42.5 \%$ of male parents and $31.5 \%$ of female parents smoked in this study sample. The profile of the participant sample in relation to teenage smoking habits, social class, and region in terms of parental smoking habits is shown in tables 1 and 2 . Approximately $50 \%$ of respondents in this survey came from nonsmoking households, approximately $10 \%$ of teenagers had a smoking mother but non-smoking father; $20 \%$ had a smoking father and nonsmoking mother, and a further $20 \%$ had two smoking parents. Among the teenagers in this study, $19 \cdot 8 \%$ girls and $16.0 \%$ boys were regular smokers and $41 \cdot 3 \%$ girls and $44 \cdot 6 \%$ boys never smokers. Both male and female teenagers from non-smoking households were significantly $(p<0.001)$ less likely to be regular smokers than teenagers from smoking households. Parents from households headed by a non-manual worker were also less likely to be smokers. Teenagers in Scotland were also significantly $(p<0.05)$ more likely, and those in the south to come from smoking households.

Initial analyses were undertaken to determine the influence of parental smoking on teenage nutrient and food intakes, allowing for differences in regional distribution, social class, and the smoking habits of the teenager, and energy intake where appropriate. Tables 3 and 4 show the unadjusted nutrient intakes of teenagers in relation to parental smoking habit for males and females respectively. No differences were noted for males or females in the intakes of energy or macronutrients in either grammes per day or when expressed as a percentage of energy. Among males, any parental smoking (that is, either or both parents smoking) was significantly associated with lower intakes of thiamin and magnesium. Intakes of vitamin $\mathrm{C}$, vitamin $\mathrm{E}$, and selenium were significantly associated with mother only smoking and both parents smoking but not by father only smoking, but the converse was true for intakes of folates. Alcohol was consumed in greater 
Table 3 The nutrient intakes of males at 16-17 years in relation to parental smoking habits

\begin{tabular}{|c|c|c|c|c|c|c|c|c|}
\hline & \multicolumn{2}{|c|}{$\begin{array}{l}\text { Neither parent smokes } \\
(n=593)\end{array}$} & \multicolumn{2}{|c|}{$\begin{array}{l}\text { Mother only smokes } \\
(n=123)\end{array}$} & \multicolumn{2}{|c|}{$\begin{array}{l}\text { Father only smokes } \\
(n=260)\end{array}$} & \multicolumn{2}{|c|}{$\begin{array}{l}\text { Both parents smoke } \\
(n=246)\end{array}$} \\
\hline & Mean & $(S E M)$ & Mean & (SEM) & Mean & (SEM) & Mean & (SEM) \\
\hline $\begin{array}{l}\text { Energy MJ } \\
\quad(\mathrm{kcal}) \\
\text { Fat }(\mathrm{g} / \mathrm{d}) \\
\text { Protein }(\mathrm{g} / \mathrm{d}) \\
\text { NMES }(\mathrm{g} / \mathrm{d}) \\
\text { IMSS }(\mathrm{g} / \mathrm{d}) \\
\text { Alcohol }(\mathrm{g} / \mathrm{d}) \\
\text { NSP }(\mathrm{g} / \mathrm{d}) \\
\text { Selenium }(\mu \mathrm{g} / \mathrm{d}) \\
\text { Magnesium }(\mathrm{mg} / \mathrm{d}) \\
\text { Carotene }(\mu \mathrm{g} / \mathrm{d}) \\
\text { Vitamin C }(\mathrm{mg} / \mathrm{d}) \\
\text { Vitamin } \mathrm{E}(\mathrm{mg} / \mathrm{d}) \\
\text { Thiamin }(\mathrm{mg} / \mathrm{d}) \\
\text { Riboflavin }(\mathrm{mg} / \mathrm{d}) \\
\text { Folates }(\mu \mathrm{g} / \mathrm{d})\end{array}$ & $\begin{array}{l}11 \cdot 6 \\
2765 \\
126 \cdot 9 \\
86 \cdot 0 \\
107 \cdot 6 \\
219 \cdot 0 \\
6 \cdot 8 \\
15 \cdot 8 \\
73 \cdot 8 \\
326 \\
2077 \\
80 \cdot 0 \\
8 \cdot 2 \\
1 \cdot 84 \\
2 \cdot 0 \\
316\end{array}$ & $\begin{array}{l}(0 \cdot 1) \\
(26) \\
(1 \cdot 3) \\
(0 \cdot 9) \\
(1 \cdot 7) \\
(2 \cdot 2) \\
(0 \cdot 5) \\
(0 \cdot 2) \\
(1 \cdot 0) \\
(4) \\
(63) \\
(2 \cdot 7) \\
(0 \cdot 1) \\
(0 \cdot \theta 2) \\
(0 \cdot 03) \\
(4)\end{array}$ & $\begin{array}{l}11 \cdot 4 \\
2374 \\
124 \cdot 9 \\
84 \cdot 5 \\
106 \cdot 3 \\
217 \cdot 8 \\
6 \cdot 2 \\
15 \cdot 0 \\
69 \cdot 4 \\
310 \\
1807 \\
69 \cdot 7 \\
7 \cdot 6 \\
1 \cdot 75 \\
2 \cdot 0 \\
306\end{array}$ & $\begin{array}{l}(0 \cdot 3) \\
(73) \\
(3 \cdot 7) \\
(2 \cdot 0) \\
(4 \cdot 0) \\
(6 \cdot 5) \\
(1 \cdot 0) \\
(0 \cdot 5) \\
(2 \cdot 4) \\
(8) \\
(121) \\
(5 \cdot 1) \\
(0 \cdot 3) \\
(0 \cdot 07) \\
(0 \cdot 09) \\
(11)\end{array}$ & $\begin{array}{l}11 \cdot 3 \\
2693 \\
124 \cdot 4 \\
84 \cdot 4 \\
105 \cdot 0 \\
213 \cdot 6 \\
5 \cdot 6 \\
15 \cdot 0 \\
71 \cdot 3 \\
316 \\
1970 \\
71 \cdot 3 \\
8 \cdot 1 \\
1 \cdot 65 \\
1 \cdot 9 \\
297\end{array}$ & $\begin{array}{l}(0 \cdot 2) \\
(38) \\
(1 \cdot 9) \\
(1 \cdot 2) \\
(2 \cdot 6) \\
(3 \cdot 5) \\
(0 \cdot 6) \\
(0 \cdot 3) \\
(1 \cdot 5) \\
(6) \\
(88) \\
(3 \cdot 5) \\
(0 \cdot 2) \\
(0.03) \\
(0.05) \\
(6)\end{array}$ & $\begin{array}{l}11 \cdot 3 \\
2693 \\
124 \cdot 5 \\
81 \cdot 8 \\
104 \cdot 0 \\
216 \cdot 2 \\
5 \cdot 4 \\
14 \cdot 8 \\
69 \cdot 3 \\
302 \\
1867 \\
61 \cdot 7 \\
7 \cdot 6 \\
1 \cdot 65 \\
1 \cdot 9 \\
293\end{array}$ & $\begin{array}{l}(0 \cdot 2) \\
(43) \\
(2 \cdot 3) \\
(1 \cdot 4) \\
(2 \cdot 6) \\
(3 \cdot 8) \\
(0 \cdot 9)^{*} \\
(0 \cdot 3) \\
(1 \cdot 7) \dagger \\
(5) \ddagger \\
(89) \\
(2 \cdot 8) \dagger \\
(0 \cdot 2) \dagger \\
(0 \cdot 04) \ddagger \\
(0 \cdot 05) \\
(7) \uparrow\end{array}$ \\
\hline
\end{tabular}

NMES $=$ non-milk extrinsic sugars. IMSS = intrinsic sugars, milk sugars and starch. NSP = non-starch polysaccharides.

* Intakes significantly lower when both parents smoke or father only smokes $(p<0 \cdot 01)$.
+ Intakes significantly lower when neither parent smokes or mother only smokes $(p<0 \cdot 05)$.

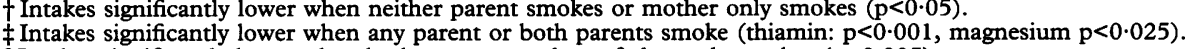

$\oint$ Intakes significantly lower when both parents smoke or father only smokes $(p<0 \cdot 005)$.

Table 4 The nutrient intakes of females at 16-17 years in relation to parental smoking habits

\begin{tabular}{|c|c|c|c|c|c|c|c|c|}
\hline & \multicolumn{2}{|c|}{$\begin{array}{l}\text { Neither parent smokes } \\
(n=809)\end{array}$} & \multicolumn{2}{|c|}{$\begin{array}{l}\text { Mother only smokes } \\
(n=174)\end{array}$} & \multicolumn{2}{|c|}{$\begin{array}{l}\text { Father only smokes } \\
(n=363)\end{array}$} & \multicolumn{2}{|c|}{$\begin{array}{l}\text { Both parents smoke } \\
(n=389)\end{array}$} \\
\hline & Mean & (SEM) & Mean & (SEM) & Mean & (SEM) & Mean & (SEM) \\
\hline \begin{tabular}{l} 
Energy MJ \\
\multicolumn{1}{c}{$(\mathrm{kcal})$} \\
Fat $(\mathrm{g} / \mathrm{d})$ \\
Protein $(\mathrm{g} / \mathrm{d})$ \\
NMES $(\mathrm{g} / \mathrm{d})$ \\
IMSS $(\mathrm{g} / \mathrm{d})$ \\
Alcohol $(\mathrm{g} / \mathrm{d})$ \\
NSP $(\mathrm{g} / \mathrm{d})$ \\
Selenium $(\mu \mathrm{g} / \mathrm{d})$ \\
Magnesium $(\mathrm{mg} / \mathrm{d})$ \\
Carotene $(\mu \mathrm{g} / \mathrm{d})$ \\
Vitamin C $(\mathrm{mg} / \mathrm{d})$ \\
Vitamin E $(\mathrm{mg} / \mathrm{d})$ \\
Thiamin $(\mathrm{mg} / \mathrm{d})$ \\
Riboflavin $(\mathrm{mg} / \mathrm{d})$ \\
Folates $(\mu \mathrm{g} / \mathrm{d})$
\end{tabular} & $\begin{array}{l}9 \cdot 0 \\
2154 \\
99 \cdot 4 \\
66 \cdot 4 \\
85 \cdot 9 \\
167 \cdot 5 \\
4 \cdot 2 \\
13 \cdot 3 \\
56 \cdot 6 \\
267 \\
2139 \\
85 \cdot 1 \\
7 \cdot 3 \\
1 \cdot 41 \\
1 \cdot 6 \\
252\end{array}$ & $\begin{array}{l}(0 \cdot 1) \\
(18) \\
(1 \cdot 0) \\
(0 \cdot 6) \\
(1 \cdot 2) \\
(1 \cdot 5) \\
(0 \cdot 3) \\
(0 \cdot 2) \\
(0 \cdot 8) \\
(3) \\
(50) \\
(2 \cdot 0) \\
(0 \cdot 1) \\
(0 \cdot 02) \\
(0 \cdot 03) \\
(3)\end{array}$ & $\begin{array}{l}8 \cdot 8 \\
2114 \\
96 \cdot 7 \\
64 \cdot 2 \\
85 \cdot 1 \\
164 \cdot 3 \\
6 \cdot 3 \\
12 \cdot 5 \\
54 \cdot 3 \\
256 \\
1828 \\
70 \cdot 8 \\
7 \cdot 2 \\
1 \cdot 34 \\
1 \cdot 5 \\
246\end{array}$ & $\begin{array}{l}(0 \cdot 2) \\
(38) \\
(2 \cdot 0) \\
(1 \cdot 2) \\
(2 \cdot 6) \\
(3 \cdot 2) \\
(0 \cdot 9) \\
(0 \cdot 3) \\
(1 \cdot 2) \\
(5) \\
(112) \\
(3 \cdot 4) \\
(0 \cdot 3) \\
(0 \cdot 04) \\
(0 \cdot 05) \\
(6)\end{array}$ & $\begin{array}{l}8 \cdot 8 \\
2113 \\
97 \cdot 5 \\
65 \cdot 7 \\
85 \cdot 6 \\
161 \cdot 8 \\
5 \cdot 0 \\
12 \cdot 4 \\
55 \cdot 4 \\
254 \\
2019 \\
81 \cdot 3 \\
6 \cdot 7 \\
1 \cdot 31 \\
1 \cdot 4 \\
239\end{array}$ & $\begin{array}{l}(0 \cdot 1) \\
(27) \\
(1 \cdot 4) \\
(0 \cdot 9) \\
(1 \cdot 8) \\
(2 \cdot 2) \\
(0 \cdot 5) \\
(0 \cdot 2) \\
(1 \cdot 0) \\
(4) \\
(76) \\
(3 \cdot 3) \\
(0 \cdot 1) \\
(0.03) \\
(0.03) \\
(5)\end{array}$ & $\begin{array}{l}8 \cdot 6 \\
2062 \\
96 \cdot 9 \\
65 \cdot 7 \\
78 \cdot 9 \\
160 \cdot 1 \\
5 \cdot 0 \\
11 \cdot 8 \\
53 \cdot 7 \\
247 \\
1760 \\
66 \cdot 1 \\
6 \cdot 4 \\
1 \cdot 31 \\
1 \cdot 3 \\
228\end{array}$ & $\begin{array}{l}(1 \cdot 1) \\
(25) \\
(1 \cdot 3)^{*} \\
(0 \cdot 9) \\
(1 \cdot 7) \\
(2 \cdot 1) \\
(0 \cdot 5) \dagger \\
(0 \cdot 2) \ddagger \\
(0 \cdot 9) \\
(5) \ddagger \\
(68) \ddagger \\
(2 \cdot 4) \Phi \\
(0 \cdot 1) \ddagger \\
(0 \cdot 04) \\
(0 \cdot 04) \ddagger \\
(5) \uparrow\end{array}$ \\
\hline
\end{tabular}

NMES = non-milk extrinsic sugars. IMSS $=$ intrinsic sugars, milk sugars and starch. NSP = non-starch polysaccharides.

* Intakes significantly lower when both parents smoke $(\mathrm{p}<0.001)$.

† Intakes significantly higher when mother only smokes $(p<0.05)$.

Intakes significantly lower when either or both parents smoke $(p<0.001)$.

Intakes significantly lower when mother only or both parents smoke $(p<0.001)$

Intakes significantly lower when father only or both parents smoke $(p<0.001)$.

quantities when parents were non-smokers or where mother only smoked. Intakes of other nutrients, although lower among males in smoking households, could be explained by either teenage smoking, social class, or regional distribution rather than by parental smoking. Among females, the intakes of NSP, vitamin $\mathrm{E}$, carotene, and riboflavin were significantly lower when there was any parental smoking in the household, but the intakes of fat and magnesium were lower only when both parents smoked. Intakes of folates were lower when father or both parents smoked, but not when mother alone smoked, and vitamin $\mathrm{C}$ intakes were lower when mother only or both parents smoked, but not when father only smoked. Alcohol intakes were higher when mother only smoked, but not when both parents smoked.

The percentage of consumers of selected food groups, and the unadjusted mean intakes in relation to consumers of each food group are shown in tables 5 and 6 for males and females respectively. Females and males in households where either or both parents smoked were less likely to be consumers of fruit juice and wholemeal bread, and more likely to be consumers of chips than those from households where neither parent smoked. Males in any smoking households were also less likely to be consumers of polyunsaturated fat spreads, cheese, and eggs. Females in any smoking households were less likely to be consumers of green vegetables, carrots, all breakfast cereals, and puddings and more likely to be consumers of non-wholemeal bread and non-polyunsaturated fat spreads compared with those in non-smoking households.

Among consumers of selected food groups, intakes of puddings, wholemeal bread, salad vegetables, and potatoes (not chips) were lower among both males and females in any smoking households. Males in households where either 
Table 5 The percentage of male consumers of selected food groups and the mean intakes of those food groups by consumers in relation to parental smoking

\begin{tabular}{|c|c|c|c|c|c|c|}
\hline \multirow[t]{2}{*}{ Food group } & \multicolumn{2}{|c|}{$\%$ consumers of food group } & \multicolumn{4}{|c|}{ Mean (SEM) intake of food group ( $g / d)$ by consumers of that food group } \\
\hline & $\begin{array}{l}\text { Neither parent } \\
\text { smokes }\end{array}$ & $\begin{array}{l}\text { Any parent } \\
\text { smokes }\end{array}$ & $\begin{array}{l}\text { Neither parent } \\
\text { smokes }\end{array}$ & $\begin{array}{l}\text { Mother only } \\
\text { smokes }\end{array}$ & $\begin{array}{l}\text { Father only } \\
\text { smokes }\end{array}$ & $\begin{array}{l}\text { Both parents } \\
\text { smoke }\end{array}$ \\
\hline Bread (not wholemeal) & $94 \cdot 9$ & $97 \cdot 3$ & $102 \cdot 9(2 \cdot 7)$ & $104 \cdot 4(6 \cdot 3)$ & $100 \cdot 2(3 \cdot 8)$ & $104.9(4 \cdot 2)$ \\
\hline Wholemeal bread & $35 \cdot 6$ & $25 \cdot 0^{*}$ & $63 \cdot 2(3 \cdot 7)$ & $47.9(7 \cdot 0)$ & $53.8(6.3)$ & $47 \cdot 4(7 \cdot 4) t$ \\
\hline Fat spreads (not polyunsaturated) & $93 \cdot 8$ & $95 \cdot 1$ & $29.9(0 \cdot 8)$ & $28 \cdot 7(1.9)$ & $27 \cdot 5(1 \cdot 1)$ & $28 \cdot 0(1 \cdot 2)$ \\
\hline Polyunsaturated fat spreads & $15 \cdot 3$ & $14 \cdot 2 \dagger$ & $19.9(1.5)$ & $17 \cdot 3(2 \cdot 3)$ & $17 \cdot 5(1.9)$ & $19 \cdot 0(2 \cdot 3)^{*}$ \\
\hline High fibre breakfast cereals & $46 \cdot 9$ & $40 \cdot 0$ & $38 \cdot 5(2 \cdot 1)$ & $37.0(5 \cdot 7)$ & $34 \cdot 3(2 \cdot 5)$ & $33.0(2 \cdot 7)$ \\
\hline Other breakfast cereals & $59 \cdot 0$ & $56 \cdot 9$ & $31.4(1.2)$ & $33 \cdot 6(3 \cdot 1)$ & $33.7(2 \cdot 6)$ & $28.4(1.6)$ \\
\hline Biscuits & $81 \cdot 3$ & $80 \cdot 9$ & $29 \cdot 8(1 \cdot 1)$ & $27 \cdot 3(2 \cdot 3)$ & $32.0(1.9)$ & $30 \cdot 2(1 \cdot 8)$ \\
\hline Puddings & $90 \cdot 0$ & $87 \cdot 1$ & $115 \cdot 5(3.3)$ & $102 \cdot 2(8 \cdot 0)$ & $100 \cdot 8(5 \cdot 1)$ & $92.9(4.5) \dagger$ \\
\hline Cheese and egg dishes & $90 \cdot 1$ & $85 \cdot 1 \dagger$ & $50.9(1.8)$ & $49.7(3.9)$ & $53 \cdot 3(2 \cdot 4)$ & $50 \cdot 7(2 \cdot 8)$ \\
\hline Meat pies & $46 \cdot 0$ & $50 \cdot 4$ & $53.9(2.2)$ & $67 \cdot 5(5 \cdot 7)$ & $57 \cdot 2(3 \cdot 4)$ & $64.4(3.8)^{*}$ \\
\hline Green vegetables & $44 \cdot 5$ & $41 \cdot 0$ & $28 \cdot 5(1 \cdot 0)$ & $28 \cdot 1(2 \cdot 4)$ & $31 \cdot 1(1 \cdot 4)$ & $25.9(1.4)$ \\
\hline Carrots & $48 \cdot 1$ & $44 \cdot 8$ & $20 \cdot 3(0 \cdot 6)$ & $20 \cdot 7(1 \cdot 6)$ & $21.9(1.2)$ & $21.9(1.2)$ \\
\hline Salad vegetables & $62 \cdot 7$ & $57 \cdot 4$ & $42 \cdot 6(1 \cdot 7)$ & $37 \cdot 5(3 \cdot 2)$ & $37 \cdot 5(2 \cdot 6)$ & $36 \cdot 0(2 \cdot 5) \dagger$ \\
\hline All vegetables & $88 \cdot 5$ & $87 \cdot 4$ & $50 \cdot 0(1.5)$ & $48 \cdot 7(3 \cdot 0)$ & $52 \cdot 2(2 \cdot 3)$ & $50 \cdot 4(2 \cdot 3)$ \\
\hline Potatoes (not chips) & $92 \cdot 1$ & $89 \cdot 3$ & $109 \cdot 2(2 \cdot 4)$ & $99 \cdot 3(5 \cdot 6)$ & $96 \cdot 1(3 \cdot 4)$ & $106.5(3.8) \dagger$ \\
\hline Chips & $75 \cdot 9$ & $86 \cdot 0 \dagger$ & $96 \cdot 4(2 \cdot 7)$ & $116 \cdot 3(6 \cdot 5)$ & $104 \cdot 8(4 \cdot 6)$ & $114.7(5.0) \dagger$ \\
\hline Fruit juice & $42 \cdot 0$ & $37 \cdot 8 \dagger$ & $171 \cdot 1(8 \cdot 5)$ & $136 \cdot 8(14 \cdot 2)$ & $136 \cdot 1(10 \cdot 1)$ & $127.4(11.5) \dagger$ \\
\hline Fruit & $73 \cdot 0$ & $65 \cdot 0$ & $86.5(3.4)$ & $88.3(8 \cdot 0)$ & $90.5(6.0)$ & $77 \cdot 4(4 \cdot 6)$ \\
\hline
\end{tabular}

* Significant difference between neither parent smoking group and either or both parents smoking groups $(\mathrm{p}<0 \cdot 01)$

+ Significant difference between neither parent smoking group and either or both parents smoking groups $(\mathrm{p}<0 \cdot 001)$.

Table 6 The percentage of female consumers of selected food groups and the mean intakes of those food groups by consumers in relation to parental smoking

\begin{tabular}{|c|c|c|c|c|c|c|}
\hline \multirow[t]{2}{*}{ Food group } & \multicolumn{2}{|c|}{$\%$ consumers of food group } & \multicolumn{4}{|c|}{ Mean (SEM) intake of food group ( $g / d)$ by consumers of that food group } \\
\hline & $\begin{array}{l}\text { Neither parent } \\
\text { smokes } \\
(n=809)\end{array}$ & $\begin{array}{l}\text { Either/both } \\
\text { parents smoke } \\
(n=926)\end{array}$ & $\begin{array}{l}\text { Neither parent } \\
\text { smokes }\end{array}$ & $\begin{array}{l}\text { Mother only } \\
\text { smokes }\end{array}$ & $\begin{array}{l}\text { Father only } \\
\text { smokes }\end{array}$ & $\begin{array}{l}\text { Bother parents } \\
\text { smoke }\end{array}$ \\
\hline Bread (not wholemeal) & $94 \cdot 2$ & $97 \cdot 3+$ & $69.4(1.5)$ & $71 \cdot 2(3 \cdot 1)$ & $68 \cdot 5(2 \cdot 1)$ & $78 \cdot 3(2 \cdot 3)^{*}$ \\
\hline Wholemeal bread & $45 \cdot 2$ & $34 \cdot 2^{*}$ & $46 \cdot 1(1.9)$ & $45 \cdot 2(4 \cdot 3)$ & $41.5(2 \cdot 8)$ & $37.6(2.5) \neq$ \\
\hline Fat spreads (not polyunsaturated) & $92 \cdot 1$ & $96.9 *$ & $20.9(0.5)$ & $20.5(0.9)$ & $20.8(0.6)$ & $21.9(0.7)$ \\
\hline Polyunsaturated fat spreads & $22 \cdot 9$ & $18 \cdot 3$ & $16.8(0.8)$ & $16 \cdot 0(2 \cdot 0)$ & $11.7(1.0)$ & $11 \cdot 2(1.0)$ \\
\hline High fibre breakfast cereals & $37 \cdot 8$ & $29 \cdot 6^{*}$ & $28 \cdot 1(1 \cdot 4)$ & $23.1(1.9)$ & $23.7(1.5)$ & $21.5(1.7)$ \\
\hline Other breakfast cereals & $43 \cdot 1$ & $37 \cdot 8+$ & $23.0(0.9)$ & $23 \cdot 8(2 \cdot 6)$ & $21 \cdot 5(1 \cdot 1)$ & $22.9(1.9)^{*}$ \\
\hline Biscuits & $84 \cdot 8$ & $81 \cdot 4$ & $24 \cdot 3(0.8)$ & $23 \cdot 4(1.4)$ & $23 \cdot 4(1 \cdot 1)$ & $19.8(0.9)$ \\
\hline Puddings & 89.9 & $86 \cdot 4 \dagger$ & $92.8(2 \cdot 4)$ & $75 \cdot 1(4 \cdot 6)$ & $81 \cdot 5(3 \cdot 2)$ & $66.6(2.9)^{*}$ \\
\hline Cheese and egg dishes & $88 \cdot 8$ & $89 \cdot 1$ & $47 \cdot 3(1 \cdot 3)$ & $43 \cdot 4(2 \cdot 3)$ & $45 \cdot 2(1.9)$ & $44.9(1.8)$ \\
\hline Meat pies & $39 \cdot 3$ & $42 \cdot 0$ & $45.6(1.7)$ & $39 \cdot 2(2 \cdot 4)$ & $42.6(1.9)$ & $46 \cdot 7(2 \cdot 2)$ \\
\hline Green vegetables & $50 \cdot 3$ & $44 \cdot 3 *$ & $27.5(0.8)$ & $27 \cdot 3(2 \cdot 2)$ & $26 \cdot 2(1 \cdot 1)$ & $25 \cdot 0(0.8)$ \\
\hline Carrots & $49 \cdot 8$ & $41.9+$ & $19 \cdot 4(0.5)$ & $23 \cdot 3(1 \cdot 7)$ & $20 \cdot 1(0.9)$ & $19 \cdot 3(0.8)$ \\
\hline Salad vegetables & $76 \cdot 5$ & 72.6 & $45.3(1.3)$ & $41 \cdot 1(3 \cdot 2)$ & $40.9(2 \cdot 0)$ & $37.0(1.6) \mathrm{t}$ \\
\hline All vegetables & $89 \cdot 4$ & $87 \cdot 3$ & $54.6(1.2)$ & $49 \cdot 9(3 \cdot 1)$ & $50.6(1.7)$ & $49 \cdot 2(2 \cdot 0)$ \\
\hline $\begin{array}{l}\text { Potatoes (not chips) }\end{array}$ & $90 \cdot 1$ & $89 \cdot 7$ & $94.0(1.9)$ & $86.7(4.0)$ & $86.9(2.7)$ & $83.6(2.5) t$ \\
\hline Chips & $66 \cdot 3$ & $75 \cdot 1 t$ & $76 \cdot 1(2 \cdot 0)$ & $85.2(4.9)$ & $80 \cdot 6(3 \cdot 1)$ & $85.9(2.9)$ \\
\hline Fruit juice & $59 \cdot 8$ & $48 \cdot 5^{*}$ & $141.6(5.2)$ & $124 \cdot 1(9 \cdot 1)$ & $146 \cdot 2(8 \cdot 2)$ & $116 \cdot 1(6 \cdot 3)$ \\
\hline Fruit & $82 \cdot 9$ & $77 \cdot 1$ & $98.9(3.0)$ & $83.1(5.4)$ & $90 \cdot 0(4 \cdot 1)$ & $84 \cdot 1(4 \cdot 0)$ \\
\hline
\end{tabular}

* Significant difference between neither parent smoking and any parents smoking groups $(p<0 \cdot 001), \dagger(p<0 \cdot 05)$.

$\ddagger$ Intakes are lower when both parents smoke only $(\mathrm{p}<0.01)$

$\ddagger$ Intakes are lower when both parents smoke only $(p<0.01)$.
Intakes are lower when mother only and both parents smoke $(p<0.005)$.

ๆI Intakes are lower when father only and both parents smoke $(p<0 \cdot 001)$.

or both parents smoked also had lower intakes of polyunsaturated fat spreads and fruit juices and higher intakes of meat pies and chips. Females in households where either or both parents smoked also had lower intakes of biscuits and higher intakes of bread that was not made from wholemeal flour. Fruit juice intakes were lower among females when mother only or both parents smoked and polyunsaturated fat spread intakes were lower for both males and females when father only smoked.

\section{Discussion}

In this study, $31.5 \%$ of mothers and $42.5 \%$ of fathers smoked. This was a slightly lower number of female and greater number of male smokers than was found in the general household survey at that time, where $35 \%$ of males and females in the 35-59 age range were smokers. ${ }^{20}$ These two groups are obviously not directly comparable, since in this study the adults were all parents of teenagers aged 16-17 years, a state which may have influenced their smoking habits. In a similar sample of New Zealand teenagers studied at a similar time, $43 \%$ of the fathers and $38 \%$ of the mothers were found to be smokers. ${ }^{21}$ It has been shown that teenagers are more likely to take up smoking if their parents are smokers ${ }^{22}$ and not surprisingly, teenagers in this study were significantly more likely to be smokers if their parents smoked, with almost a third of girls and a quarter of boys smoking as teenagers if both their parents smoked. Since teenage smoking has been shown to influence teenage dietary habits, ${ }^{6}$ this was an important covariate when examining the potential influence of parental smoking on teenage diet. A number of recent studies have examined the smoking habits of young people in Britain, and the Health Education Authority (HEA) reported that $17 \%$ of 16 year old females and $19 \%$ of 16 year old males were regular smokers, rising to $24 \%$ and $22 \%$ of 17 year old males and females respectively. ${ }^{23}$ Other studies have also shown 
that girls are more likely to be smokers than boys at 17 years $^{24}$ and this was also found in the data reported here, where $16.0 \%$ of males and $19.8 \%$ of females claimed to be regular smokers.

While dietary recording periods of more than seven days are generally necessary for many micronutrients in order to classify individuals into extremes of intake, the collection of data over three or four days has been suggested as an adequate time period when an average nutrient intake for a group of individuals is required. ${ }^{25}$ Nutrient intakes for teenagers in this study were comparable with data collected elsewhere for this population group. ${ }^{12}$ The role of parental smoking on teenage dietary intakes appeared in many ways to be consistent with both those dietary differences observed between smoking and non-smoking teenagers, and with those previously reported among smoking adults. Those nutrients which were generally lower in the diets of teenagers when their parents smoked were NSP, vitamins $C$ and $\mathrm{E}$, carotene, folates, and magnesium. These nutrients are highly associated with intakes of fruit, fruit juice, vegetables, cereals, and polyunsaturated fat spreads and not surprisingly, teenagers in households where there was a smoking parent were both less likely to consume these foods and to consume smaller amounts than those in households where neither parent smoked. Teenagers from smoking households were also more likely to consume chips and non-wholemeal bread, and to consume larger amounts of these foods than those in non-smoking households.

Many studies of adult smokers have shown a positive association between smoking and alcohol consumption ${ }^{124}$ and this has also been reported recently among teenage smokers. ${ }^{624}$ It might be expected that teenagers of smoking parents drink more alcohol than those of nonsmoking parents, but in this study teenagers in households where father only or both parents smoked did not report increased alcohol intakes. Significantly greater intakes of alcohol were reported among males from non-smoking households and among both males and females of smoking mothers, allowing for differences in teenage smoking, social class, and region. Since smoking mothers were likely to be consuming more alcohol than non-smoking mothers, it is not possible from this study to determine whether the alcohol intake of teenagers is associated with maternal smoking per se or with the probable positively associated maternal alcohol consumption.

Among both males and females, vitamin C intakes were associated with maternal rather than paternal smoking, and this seemed to be related to fruit juice intakes among females but not among males. Since the intake of fruit juice is generally higher among females, the relative difference in intakes between smoking and nonsmoking mothers may have been more significant than among smoking and non-smoking fathers. The higher intake of alcoholic beverages and lower fruit juice consumption among the daughters of smoking mothers may also be related. Folate intakes were associated with paternal rather than maternal smoking among both males and females, but this was not explained by any particular difference in food choice by the teenagers of smoking fathers. Intakes of polyunsaturated fat spreads were significantly associated with paternal but not maternal smoking patterns among both males and females, suggesting that the choice of fat spread in the house may have been determined by father, rather than mother's preference.

It has been suggested that smokers are at risk from chronic diseases not only because they are more susceptible to oxidative tissue damage from tobacco smoke, but because they consume diets which are also lower in protective antioxidant nutrients. ${ }^{1}$ Whilst it has been shown that adult and teenage smokers consume diets significantly lower in antioxidant nutrients, particularly those from fruit, fruit juices, and vegetables, than non-smokers, it also appears that teenagers whose diets are in part chosen by smoking parents have similar dietary patterns. It has been reported that passive smoking by teenagers can both influence their blood lipid levels, potentially increasing their risk of premature cardiovascular disease, and increase their risk of lung cancer in later life. ${ }^{26}$ Smokers have also been shown to have increased requirements for a number of micronutrients, particularly vitamin $\mathrm{C}^{27}$ and it is possible that non-smoking teenagers in smoking households may have a similar increased requirement. The poorer diets chosen by smoking parents are therefore likely to exacerbate the damage caused to these passive smokers. The diets that these teenagers are exposed to as adolescents may also influence the dietary habits that they themselves take into adulthood. It has been reported elsewhere that food preferences appear to be consolidated during childhood, and that although young people's behaviour changes over time the relative ranking of individuals in terms of healthy food choices remains similar. ${ }^{28}$ Children who have grown up with smoking parents may therefore have been initiated into health-compromising behaviours that they will maintain relative to their peers from non-smoking environments. Since it has been suggested that diets lower in wholegrain cereals, fruits, vegetables, and polyunsaturated fat spreads may offer less protection against chronic disease among the population as a whole, ${ }^{29}$ it can be suggested that the teenagers of smoking parents are doubly disadvantaged by their exposure to tobacco smoke and poor dietary habits.

This study was supported by a grant from The British Heart Foundation.

1 Margetts BA, Jackson AA. Interactions between people's diet and their smoking habits: The dietary and nutritional survey of British adults. BMF 1993;307:1381-84.

2 Bolton-Smith C, Woodward M, Brown CA, Smith WCS Tunstall-Pedoe $\mathrm{H}$. Nutrient intakes from current, ex- and never smokers: results from the Scottish heart health study. Proc Nutr Soc 1991;50:36A.

3 Cade J, Margetts B. Smoking and diet: is the diet of smokers different? Proc Nutr Soc 1990;49:41A

4 Whichelow MJ, Erzinclioglu SW. Comparison of the diets of smokers and non-smokers. Proc Nutr Soc 1990;49:42A

5 Fehily AM, Phillips KM, Yarnell JWG. Diet, smoking, social class and body mass index in the Caerphilly heart diseas study. Am f Clin Nutr 1984;40:827-33. 
6 Crawley HF, While D. The dietary habits and body weight of British teenage smokers aged 16-17 years. Eur $\mathcal{F}$ Clin Nutr 1995;49:904-14.

7 Prättälä R. Young people and food: socio-cultural studies of food consumption patterns. Helsinki: University Helsinki, 1989. consumption

8 Murcott A. ed. The sociology of food and eating. Aldershot: Gower, 1983.

9 Chamberlain R, Chamberlain G, Howlett B, Claireaux. British births 1970. Vol. 1: The first week of life. London: Heinemann Medical Books Ltd, 1975.

10 Ekinsmyth C, Bynner J, Montgomery S, Shepherd P. An integrated approach to the design and analysis of the 1970 British cohort study (BCS70) and the national child development study (NCDS). Inter-cohort analysis working papers, 1. SSRU 1992. London: City University, 1992.

11 OPCS. Regional trends. London: HMSO, 1988

12 Crawley HF. The energy, nutrient and food intakes of teenagers aged 16-17 years in Britain. 1. Energy, macronutrients and non-starch polysaccharides. Br f Nutr 1993; 70:15-26.

13 Crawley HF. Food portion sizes. London: HMSO, 1988.

13 Crawley HF. Food portion sizes. London: HMSO, 1988. considerations on dietary assessment methods. Eur $\mathcal{f}$ Clin Nutr 1988;43:453-63.

15 Black AE, Goldberg GR, Jebb SA, Livingstone MBE, Cole TJ, Prentice AM. Critical evaluation of energy intake data using fundamental principles of energy physiology: 2 . Evaluating the results of published surveys. Eur $\mathcal{F}$ Clin Nutr 1991;45:583-99.

16 Beverley BR, Day I, Ide L. Smoking in children in Great Britain. London: Social Sciences Research Council/Medical Research Council, 1972.
17 Thomson M, Holroyd S, Goddard E. Smoking among secondary schoolchildren in 1992. London: OPCS, 1993.

18 Payne CD. The GLIM system: release 3.77. Oxford: National Algorithms Group, 1985.

19 SPSS Inc. SPSS-X user's guide. 4th ed. Chicago: SPSS, 1990.

20 OPCS. The general household survey. London: OPCS, 1989

21 Stanton WR, Silva PA. Tracking changes in the patterns of parental smoking. $\mathcal{F}$ Roy Soc Health February 1993; 12-15.

22 Pearson R, Richardson K. The smoking habits of 16 yea olds in the national child development study. Public Health 1978;92:136-44.

23 Health Education Authority. Today's young adults: 16-19 year olds look at diet, alcohol, smoking, drugs and sexual behaviour. London: HEA, 1992.

24 Townsend J, Wilkes $\mathrm{H}$, Haines A, Jarvis M. Adolescen smokers seen in general practice: health, lifestyle, physical measurements, and response to anti-smoking advice. $B M F$ 1991;303:947-50.

25 Bingham $S$. The dietary assessment of individuals: methods, acuracy, new techniques and recommendations. Nutr Abstr acuracy, new techniques

26 Charlton A. Children and passive smoking: A review. $\mathcal{f}$ Fam Pract 1994;38:267-77.

27 Kallner AB, Hartmann D, Hornig D. On the requirements of ascorbic acid in man: steady state turnover and body pool. Am F Clin Nutr 1981;34:1347-5

28 Kelder JH, Perry CL, Klepp KI, Lytle LL. Longitudina tracking of adolescent smoking, physical activity and foo choice behaviours. Am $\mathcal{f}$ Public Health 1994;84:1121-26.

29 World Health Organisation. Diet, nutrition and chronic diseases. Technical Report Series no. 797. Geneva: WHO 1990. 